PROCEEDINGS OF THE

AMERICAN MATHEMATICAL SOCIETY

Volume 128, Number 7, Pages 2033-2042

S 0002-9939(99)05369-1

Article electronically published on November 23, 1999

\title{
JOINT MEAN OSCILLATION AND LOCAL IDEALS IN THE TOEPLITZ ALGEBRA
}

\author{
JINGBO XIA
}

(Communicated by David R. Larson)

\begin{abstract}
We introduce the joint local mean oscillation $\operatorname{LMO}(f, g)(\tau)$ and discuss to what extent this function-theoretical quantity serves as a $C^{*}$-algebraic invariant in the full Toeplitz algebra $\mathcal{T}=\mathcal{T}\left(L^{\infty}\right)$.
\end{abstract}

\section{INTRODUCTION}

Throughout the paper, $T$ denotes the unit circle and $d m$ the Lebesgue measure on $T$ normalized so that $m(T)=1$. We write $L^{p}$ for $L^{p}(T, d m)$ and $H^{p}$ for the Hardy subspace of $L^{p}, 1 \leq p \leq \infty$. Let $P: L^{2} \rightarrow H^{2}$ be the orthogonal projection. Given $f \in L^{\infty}$, the Toeplitz operator $T_{f}$ and the Hankel operator $H_{f}$ are defined by the formulas $T_{f} \varphi=\operatorname{Pf} \varphi$ and $H_{f} \varphi=(1-P) f \varphi$ respectively, $\varphi \in H^{2}$. We have $T_{\bar{g} f}-T_{\bar{g}} T_{f}=H_{g}^{*} H_{f}$. Let $\mathcal{T}$ denote the full Toeplitz algebra. That is, $\mathcal{T}$ is the $C^{*}$-algebra generated by $\left\{T_{f}: f \in L^{\infty}\right\}$. Let $\mathcal{K}$ be the collection of compact operators on $H^{2}$. It is well known that $\mathcal{K} \subset \mathcal{T}$.

Recall that, if $f \in L^{\infty}$ is a real-valued function, then the well-known theorem of Sarason [5] tells us that the operator $H_{f}$ is compact (equivalently, $T_{f^{2}}-T_{f}^{2}=H_{f}^{*} H_{f}$ is compact) if and only if $f \in \mathrm{VMO}$. Using the localization in $\mathcal{T}$, this result was quantitatively refined and extended in [4 to cover $H_{f}$ which is only "partially" compact or not compact at all.

For each $\tau \in T$, let $\mathcal{K}_{\tau}$ denote the ideal in $\mathcal{T}$ generated by $\mathcal{K}$ and $\left\{T_{\eta}: \eta \in C(T)\right.$, $\eta(\tau)=0\}$. Let $\Phi_{\tau}: \mathcal{T} \rightarrow \mathcal{T} / \mathcal{K}_{\tau}$ be the quotient homomorphism. Recall that the usual localization in $\mathcal{T}$ is simply the fact that $\bigcap_{\tau \in T} \mathcal{K}_{\tau}=\mathcal{K}$ [2]. Equivalently, for any $A \in \mathcal{T}$, we have $\inf \{\|A+K\|: K \in \mathcal{K}\}=\sup _{\tau \in T}\left\|\Phi_{\tau}(A)\right\|$. Thus $\left\|\Phi_{\tau}(A)\right\|$ is the local distance of $A$ from $\mathcal{K}$. In [4, this was determined in terms of functiontheoretical data in the case $A=T_{f^{2}}-T_{f}^{2}=H_{f}^{*} H_{f}$. For $f \in \mathrm{BMO}$ and $\tau \in T$, the local mean oscillation of $f$ at $\tau$ is

$$
\operatorname{LMO}(f)(\tau)=\lim _{\delta \downarrow 0} \sup \left\{\frac{1}{|I|} \int_{I}\left|f-f_{I}\right| d m:|\lambda-\tau| \leq \delta \text { for all } \lambda \in I\right\} .
$$

Here and in what follows, $I$ always denotes an arc in $T$ with $|I|=m(I)>0$, and $f_{I}=\int_{I} f d m /|I|$. The following is the refinement of Sarason's theorem mentioned earlier.

Received by the editors August 24, 1998.

1991 Mathematics Subject Classification. Primary 46H10, 47B35, 47C15.

This research was supported in part by National Science Foundation grant DMS-9703515. 
Theorem 1 ([4, Theorems 5.4 and 5.5]). (i) There exist absolute constants $C>$ $c>0$ such that $c L M O(f)(\tau) \leq\left\|\Phi_{\tau}\left(H_{f}^{*} H_{f}\right)\right\|^{1 / 2} \leq C L O M(f)(\tau)$ for all real-valued $f \in L^{\infty}$ and all $\tau \in T$.

(ii) Consequently, for real-valued $f \in L^{\infty}, H_{f}^{*} H_{f} \in \mathcal{K}_{\tau}$ if and only if $\operatorname{LMO}(f)(\tau)$ $=0$.

This makes $\operatorname{LMO}(f)(\tau)$ a useful invariant in the study of certain automorphisms of $\mathcal{T}$ [4]. Obviously one would like to generalize the above to the case where there are two symbol functions involved, i.e., to operators $H_{g}^{*} H_{f}$. By the works of Axler, Chang, Sarason [1] and Volberg [7], $H_{g}^{*} H_{f} \in \mathcal{K}$ if and only if $H^{\infty}[f] \cap H^{\infty}[g] \subset$ $H^{\infty}+C(T)$. (Also see [8], 10, [1].) But it is still important to have a description of the local distance $\left\|\Phi_{\tau}\left(H_{g}^{*} H_{f}\right)\right\|$ in terms of local oscillation. The purpose of this note is to report that, somewhat to our surprise, the case of two symbols turns out to be different. That is, while the analogue of Theorem 1(ii) still holds true, that of Theorem 1(i) does not.

Given $f, g \in \mathrm{BMO}$ and $\tau \in T$, define

$$
\begin{aligned}
& \operatorname{LMO}(f, g)(\tau)=\lim _{\delta \downarrow 0} \sup \left\{\frac{1}{|I|} \int_{I}\left|f-f_{I}\right| d m \frac{1}{|I|} \int_{I}\left|g-g_{I}\right| d m:\right. \\
& \qquad|\lambda-\tau| \leq \delta \text { for all } \lambda \in I\},
\end{aligned}
$$

which we call the joint local mean oscillation of $f$ and $g$ at $\tau$.

Recall that if $f \in \mathrm{BMO}$, then there are $u, v \in L^{\infty}$ such that $f=u+P v$. This implies that $H_{g}^{*} H_{f} \in \mathcal{T}$ if $f, g \in$ BMO. Throughout the paper, we let $Q=1-P$.

Theorem 2. (a) Let $\tau \in T$ and $f, g \in B M O$. Then $H_{g}^{*} H_{f} \in \mathcal{K}_{\tau}$ if and only if $L M O(Q f, Q g)(\tau)=0$.

(b) For $\tau \in T$ and real-valued $f, g \in B M O, H_{g}^{*} H_{f} \in \mathcal{K}_{\tau}$ if and only if $L M O(f, g)(\tau)=0$.

Because $\mathcal{K}=\bigcap_{\tau \in T} \mathcal{K}_{\tau}$, an immediate by-product of this theorem is

Corollary 3. (a) For any $f, g \in B M O, H_{g}^{*} H_{f} \in \mathcal{K}$ if and only if

$$
\lim _{|I| \downarrow 0} \frac{1}{|I|} \int_{I}\left|Q f-(Q f)_{I}\right| d m \frac{1}{|I|} \int_{I}\left|Q g-(Q g)_{I}\right| d m=0 .
$$

(b) For any real-valued $f, g \in B M O, H_{g}^{*} H_{f} \in \mathcal{K}$ if and only if

$$
\lim _{|I| \downarrow 0} \frac{1}{|I|} \int_{I}\left|f-f_{I}\right| d m \frac{1}{|I|} \int_{I}\left|g-g_{I}\right| d m=0 .
$$

Compare this with the existing results on the compactness of $H_{g}^{*} H_{f}$.

Proposition 4. $L M O(f, g)(\tau)$ fails to dominate $\left\|\Phi_{\tau}\left(H_{g}^{*} H_{f}\right)\right\|$. More specifically, given any $\epsilon>0$, there are real-valued $f, g \in L^{\infty}$ such that $0<\operatorname{LMO}(f, g)(1) \leq$ $\epsilon\left\|\Phi_{1}\left(H_{g}^{*} H_{f}\right)\right\|$.

Even though $\operatorname{LMO}(f, g)(\tau)$ does not in general dominate $\left\|\Phi_{\tau}\left(H_{g}^{*} H_{f}\right)\right\|$, there is an upper bound for this local distance in terms of a joint oscillation involving the Poisson kernel. Denote $P_{z}(\tau)=\left(1-|z|^{2}\right) /|\tau-z|^{2},|z|<1$ and $\tau \in T$. For $f \in L^{1}$, we let $f(z)=\int_{T} f P_{z} d m$. For $1 \leq p<\infty, f, g \in$ BMO and $\tau \in T$, define

$$
\begin{array}{r}
P_{p}(f, g ; \tau)=\limsup _{\delta \downarrow 0}\left\{\left(\int_{T}|f-f(z)|^{p} P_{z} d m \int_{T}|g-g(z)|^{p} P_{z} d m\right)^{1 / p}:\right. \\
|z|<1,|z-\tau| \leq \delta\},
\end{array}
$$




$$
\begin{aligned}
M_{p}(f, g ; \tau)=\lim _{\delta \downarrow 0} \sup \left\{\left(\frac{1}{|I|} \int_{I}\left|f-f_{I}\right|^{p} d m \frac{1}{|I|} \int_{I}\left|g-g_{I}\right|^{p} d m\right)^{1 / p}:\right. & \\
|\lambda-\tau| \leq \delta & \text { for } \text { all } \lambda \in I\} .
\end{aligned}
$$

Thus $\operatorname{LMO}(f, g)(\tau)=M_{1}(f, g ; \tau)$.

Theorem 5. For each $2<p<\infty$, there is a constant $C(p)>0$ such that $\left\|\Phi_{\tau}\left(H_{g}^{*} H_{f}\right)\right\| \leq C(p) P_{p}(f, g ; \tau)$ for all $f, g \in B M O$ and $\tau \in T$.

The construction in Section 3 shows that, given $1 \leq p^{\prime}<p<\infty$ and $\tau \in T$, the set $\left\{M_{p}(f, g ; \tau) / M_{p^{\prime}}(f, g ; \tau): f, g \in L^{\infty}, M_{p^{\prime}}(f, g ; \tau)>0\right\}$ becomes unbounded as a result of the collaboration between dissimilar $f$ and $g$, which is in sharp contrast with [4, Theorem 5.5]. The reason for this is that the John-Nirenberg theorem cannot be applied to the joint oscillation of two BMO functions. In other words, the quantities $M_{p}(f, g ; \tau)$ and $M_{p^{\prime}}(f, g ; \tau)$ are generally not comparable. Therefore it is not a trivial fact that they vanish simultaneously.

Theorem 6. Given $f, g \in B M O$ and $\tau \in T$, the following are equivalent:

(a) $M_{1}(f, g ; \tau)=0$.

(b) $M_{p}(f, g ; \tau)=0$ for every $p \in[1, \infty)$.

(c) $P_{1}(f, g ; \tau)=0$.

(d) $P_{p}(f, g ; \tau)=0$ for every $p \in[1, \infty)$.

The rest of the paper consists of the proofs of these results; Theorems 2, 5 and 6 will be proved in Section 2 and the proof of Proposition 4 will be given in Section 3.

\section{Local estimates}

The proof of Theorem 5 relies on a number of ideas and results from [4], [9, [10, which we will now recall. Denote the unit disc $\{z \in \mathbf{C}:|z|<1\}$ by $D$. For each $\tau \in T$, let $\Gamma_{\tau}=\{z \in D:|\tau-z|<2(1-|z|), 3 / 4<|z|<1\}$. In what follows $F$ and $G$ will always be $L^{2}$-valued functions on $D$ which are continuous with respect to the norm topology. To avoid confusion with complex-valued functions, the values of $F$ and $G$ at $z \in D$ will be denoted by $F_{z}$ and $G_{z}$ respectively. In other words, for any $z \in D, F_{z}$ and $G_{z}$ are themselves functions on $T$. Recall from [9] that, for any $\varphi, \psi \in L^{2}$, the rigged non-tangential maximal function $M_{F, G}(\varphi, \psi)$ is defined as

$$
M_{F, G}(\varphi, \psi)(\tau)=\sup _{z \in \Gamma_{\tau}} \int_{T}\left|F_{z} \varphi\right| P_{z} d m \int_{T}\left|G_{z} \psi\right| P_{z} d m, \quad \tau \in T
$$

Proof of Theorem 5. For each $0 \leq r<1$, let $u_{r}(\tau)=(1-r) /(1-r \tau)$. Then $u_{r} \in H^{\infty}, u_{r}(1)=1,\left\|u_{r}\right\|_{\infty}=1$, and $\left\|u_{r}\right\|_{2}=\sqrt{(1-r) /(1+r)}$. We first show that there are $C_{1}>0$ and $C_{2}>0$ such that for any $f, g \in \operatorname{BMO}, \varphi, \psi \in H^{2}$ and $0 \leq r<1$

$$
\left|\left\langle H_{f} u_{r} \varphi, H_{g} u_{r} \psi\right\rangle\right| \leq C_{1}\left\|M_{F, G}\left(u_{r} \varphi, u_{r} \psi\right)\right\|_{1}+C_{2}\|f\|_{4}\|g\|_{4} \sqrt{1-r}\|\varphi\|_{2}\|\psi\|_{2},
$$

where $F$ and $G$ are such that $F_{z}=f-f(z)$ and $G_{z}=g-g(z), z \in D$. 
Since the harmonic extensions of $H_{f} u_{r} \varphi$ and $H_{g} u_{r} \psi$ vanish at $z=0$, it follows from the Littlewood-Paley formula (see, e.g., page 236 of [3]) that

$$
\begin{aligned}
\left|\left\langle H_{f} u_{r} \varphi, H_{g} u_{r} \psi\right\rangle\right| & \leq \frac{1}{\pi} \int_{D}\left|\left\langle\nabla\left(H_{f} u_{r} \varphi\right)(z), \nabla\left(H_{g} u_{r} \psi\right)(z)\right\rangle_{\mathbf{C}^{2}}\right| \log \frac{1}{|z|} d A(z) \\
& =\frac{1}{\pi} \int_{7 / 8<|z|<1}+\frac{1}{\pi} \int_{|z| \leq 7 / 8} .
\end{aligned}
$$

It was shown in the proof of [9, Proposition 2.2] that there is a $C_{1}>0$ such that

$$
\begin{aligned}
& \frac{1}{\pi} \int_{7 / 8<|z|<1}\left|\left\langle\nabla\left(H_{f} u_{r} \varphi\right)(z), \nabla\left(H_{g} u_{r} \psi\right)(z)\right\rangle_{\mathbf{C}^{2}}\right| \log \frac{1}{|z|} d A(z) \\
& \quad \leq C_{1}\left\|M_{F, G}\left(u_{r} \varphi, u_{r} \psi\right)\right\|_{1} .
\end{aligned}
$$

To estimate $\frac{1}{\pi} \int_{|z| \leq 7 / 8}$, note that $\sup _{|z| \leq 7 / 8} \sup _{\tau \in T}\left|\bar{\partial}_{z} P_{z}(\tau)\right|=64$ since $\bar{\partial}_{z} P_{z}(\tau)=$ $(1-\bar{z} \tau)^{-2} \tau$. Since $\partial_{z}\left(H_{f} u_{r} \varphi\right)(z)=0$ and $\bar{\partial}_{z}\left(H_{f} u_{r} \varphi\right)(z)=\bar{\partial}_{z}\left(f u_{r} \varphi\right)(z)$, when $|z| \leq 7 / 8$

$$
\begin{aligned}
\left|\nabla\left(H_{f} u_{r} \varphi\right)(z)\right| & =\sqrt{2}\left|\bar{\partial}_{z} \int_{T} f u_{r} \varphi P_{z} d m\right| \leq 64 \sqrt{2}\left\|f u_{r}\right\|_{2}\|\varphi\|_{2} \\
& \leq 64 \sqrt{2}\|f\|_{4}(1-r)^{1 / 4}\|\varphi\|_{2} .
\end{aligned}
$$

And a similar estimate holds for $\left|\nabla\left(H_{g} u_{r} \psi\right)(z)\right|$. To complete the proof of (2.1), it now suffices to remark that $\log |z|^{-1} d A(z)$ is a finite measure on $\{z:|z| \leq 7 / 8\}$.

We next show that, for each $p>2$, there is a $C_{3}(p)>0$ such that the following holds true: For any $\epsilon>0$, there is a $\rho=\rho(\epsilon, p, f, g) \in(0,1)$ such that

$$
\left\|M_{F, G}\left(u_{r} \varphi, u_{r} \psi\right)\right\|_{1} \leq C_{3}(p)\left\{P_{p}(f, g ; 1)+\epsilon\right\}\|\varphi\|_{2}\|\psi\|_{2},
$$

if $\rho \leq r<1$ and $\varphi, \psi \in H^{2}$. For each $t \in[1, \infty)$, there is a $B_{t}>0$ such that

$$
\sup _{z \in D}\left(\int_{T}|h-h(z)|^{t} P_{z} d m\right)^{1 / t} \leq B_{t}\|h\|_{\mathrm{BMO}}, \quad h \in \mathrm{BMO} .
$$

See, e.g., [3, Chapter VI]. Let $0<\alpha<1$ be such that $\alpha+(2 \alpha)^{1 / p} B_{2 p}^{2}\|f\|_{\text {BMO }}\|g\|_{\text {BMO }}$ $\leq \epsilon$. It follows from the definition of $P_{p}(f, g ; 1)$ that there is a $\delta=\delta(p, f, g) \in(0,1)$ such that

$$
\left(\int_{T}\left|F_{z}\right|^{p} P_{z} d m \int_{T}\left|G_{z}\right|^{p} P_{z} d m\right)^{1 / p} \leq \alpha+P_{p}(f, g ; 1) \text { if }|1-z| \leq \delta \text { and } z \in D .
$$

Let $J$ be the open arc in $T$ whose center is 1 with $|J|=\alpha \delta^{2} / 8$. It is easy to see that there is a $\rho=\rho(\delta, \alpha) \in(0,1)$ such that $\sup _{\tau \in T \backslash J}\left|u_{r}(\tau)\right| \leq \alpha$ if $\rho \leq$ $r<1$. Thus if $\rho \leq r<1,|1-z| \geq \delta$ and $z \in D$, then $\left(\int\left|F_{z} u_{r}\right|^{p} P_{z} d m\right)^{1 / p} \leq$ $\left(\int\left|u_{r}\right| P_{z} d m\right)^{1 / 2 p} B_{2 p}\|f\|_{\mathrm{BMO}}$ and

$$
\begin{aligned}
\int_{T}\left|u_{r}\right| P_{z} d m & =\int_{T \backslash J}\left|u_{r}\right| P_{z} d m+\int_{J}\left|u_{r}\right| P_{z} d m \leq \alpha+|J| \sup _{\tau \in J}|\tau-z|^{-2} \\
& \leq \alpha+\left(\alpha \delta^{2} / 8\right)(|1-z|-\pi|J|)^{-2} \leq 2 \alpha .
\end{aligned}
$$

Hence, if we write $W(z, r)=\left(\int_{T}\left|F_{z} u_{r}\right|^{p} P_{z} d m \int_{T}\left|G_{z} u_{r}\right|^{p} P_{z} d m\right)^{1 / p}$, then

$$
W(z, r) \leq \max \left\{\alpha+P_{p}(f, g ; 1),(2 \alpha)^{1 / p} B_{2 p}^{2}\|f\|_{\mathrm{BMO}}\|g\|_{\mathrm{BMO}}\right\} \leq P_{p}(f, g ; 1)+\epsilon
$$


for every $z \in D$ if $\rho \leq r<1$. Let $q=p /(p-1)$. Now, if $\rho \leq r<1$, then

$$
\begin{aligned}
M_{F, G}\left(u_{r} \varphi, u_{r} \psi\right)(\tau) & \leq \sup _{z \in \Gamma_{\tau}} W(z, r)\left(\int_{T}|\varphi|^{q} P_{z} d m \int_{T}|\psi|^{q} P_{z} d m\right)^{1 / q} \\
& \leq\left\{P_{p}(f, g ; 1)+\epsilon\right\}\left(M_{\mathrm{nt}}\left(|\varphi|^{q}\right)(\tau) M_{\mathrm{nt}}\left(|\psi|^{q}\right)(\tau)\right)^{1 / q},
\end{aligned}
$$

where $M_{\text {nt }}$ denotes the non-tangential maximal operator. Since $1<q<2$ and $|\varphi|^{q} \in L^{2 / q}, \int_{T}\left\{M_{\mathrm{nt}}\left(|\varphi|^{q}\right)\right\}^{2 / q} d m \leq C_{3}(p) \int_{T}\left\{|\varphi|^{q}\right\}^{2 / q} d m=C_{3}(p)\|\varphi\|_{2}^{2}$ by the wellknown properties of $M_{\mathrm{nt}}$, where $C_{3}(p)>0$ depends only on $p$ (see [3, page 24]). This proves (2.2).

By the obvious circular symmetry, it suffices to prove the theorem for the point $\tau=1$ in $T$. By (2.1) and (2.2), there is a $C(p)>0$ which depends only on $p>2$ such that the following holds true: For any $\eta>0$, there is an $r_{0}=r_{0}(p, f, g, \eta) \in(0,1)$ such that

$$
\left|\left\langle H_{f} u_{r} \varphi, H_{g} u_{r} \psi\right\rangle\right| \leq C(p)\left\{P_{p}(f, g ; 1)+\eta\right\}\|\varphi\|_{2}\|\psi\|_{2} \text { if } r_{0} \leq r<1 \text { and } \varphi, \psi \in H^{2} \text {. }
$$

That is, $\left\|T_{\bar{u}_{r}} H_{g}^{*} H_{f} T_{u_{r}}\right\| \leq C(p)\left(P_{p}(f, g ; 1)+\eta\right)$ if $r_{0} \leq r<1$. Since $u_{r} \in C(T)$ and $u_{r}(1)=1$, we have $\Phi_{1}\left(T_{\bar{u}_{r}} H_{g}^{*} H_{f} T_{u_{r}}\right)=\Phi_{1}\left(H_{g}^{*} H_{f}\right)$. Thus $\left\|\Phi_{1}\left(H_{g}^{*} H_{f}\right)\right\| \leq$ $C(p)\left(P_{p}(f, g ; 1)+\eta\right)$. Because $\eta>0$ is arbitrary, this concludes the proof.

For each $z \in D$, define the inner function $\xi_{z}(\tau)=(z-\tau) /(1-\bar{z} \tau)$.

Lemma 7. For any $\tau_{0} \in T$ and $A \in \mathcal{K}_{\tau_{0}}$, we have $\lim _{z \rightarrow \tau_{0},|z|<1}\left\|A-T_{\xi_{z}}^{*} A T_{\xi_{z}}\right\|=0$.

Proof. Lemma 2 of [10] tells us that

$$
\lim _{|z| \uparrow 1}\left\|K-T_{\xi_{z}}^{*} K T_{\xi_{z}}\right\|=0 \text { if } K \in \mathcal{K} .
$$

By the structure of $\mathcal{K}_{\tau_{0}}$, therefore, it suffices to prove the lemma in the case where $A=T_{\eta} T_{g_{1}} \ldots T_{g_{n}}, g_{1}, \ldots, g_{n} \in L^{\infty}$, and $\eta \in C(T)$ with $\eta=0$ in a neighborhood of $\tau_{0}$. We can write $\eta=\eta_{1} \ldots \eta_{n}$, where every $\eta_{j}$ is continuous on $T$ and vanishes in a neighborhood of $\tau_{0}$. (For example, let $\eta_{2}=\ldots=\eta_{n}=|\eta|^{1 / n}$ and $\eta_{1}(\tau)=$ $|\eta(\tau)|^{(1-n) / n} \eta(\tau)$ when $\eta(\tau) \neq 0$ and $\eta_{1}(\tau)=0$ when $\eta(\tau)=0$.) Thus, if we set $B=T_{\eta_{1} g_{1} \ldots T_{\eta_{n} g_{n}}}$, then $A-B \in \mathcal{K}$. By (2.4), the proof of the lemma is reduced to that of

$$
\lim _{\substack{z \rightarrow \tau_{0} \\|z|<1}}\left\|B-T_{\xi_{z}}^{*} B T_{\xi_{z}}\right\|=0 .
$$

We use induction on $n$. Since $T_{\xi_{z}}^{*} T_{f} T_{\xi_{z}}=T_{f\left|\xi_{z}\right|^{2}}=T_{f}$, (2.5) certainly holds when $n=1$. Write $B=T_{\eta_{1} g_{1}} B^{\prime}$. We have

$$
B-T_{\xi_{z}}^{*} B T_{\xi_{z}}=\left\{T_{\eta_{1} g_{1}} B^{\prime}-T_{\xi_{z}}^{*} T_{\eta_{1} g_{1}} T_{\xi_{z}} T_{\xi_{z}}^{*} B^{\prime} T_{\xi_{z}}\right\}-T_{\xi_{z}}^{*} T_{\eta_{1} g_{1}}\left(1-T_{\xi_{z}} T_{\xi_{z}}^{*}\right) B^{\prime} T_{\xi_{z}} .
$$

By the induction hypothesis, the norm of the term $\{\ldots\}$ above tends to 0 as $z \rightarrow \tau_{0}$ in $D$.

Now $1-T_{\xi_{z}} T_{\xi_{z}}^{*}=k_{z} \otimes k_{z}$, where $k_{z}(\tau)=\left(1-|z|^{2}\right)^{1 / 2} /(1-\bar{z} \tau)$ [10, page 480]. Thus

$$
\left\|T_{\xi_{z}}^{*} T_{\eta_{1} g_{1}}\left(1-T_{\xi_{z}} T_{\xi_{z}}^{*}\right) B^{\prime} T_{\xi_{z}}\right\| \leq\left\|B^{\prime} T_{\xi_{z}}\right\|\left\|T_{\xi_{z}}^{*} T_{\eta_{1} g_{1}} k_{z}\right\|_{2}
$$


Suppose that $\delta>0$ is such that $\eta_{1}(\tau)=0$ if $\left|\tau-\tau_{0}\right| \leq \delta$. For $z \in D$ and $\tau \in T$ such that $\left|z-\tau_{0}\right| \leq \delta / 2$ and $\left|\tau-\tau_{0}\right|>\delta$, we have

$$
\left|k_{z}(\tau)\right|=\frac{\sqrt{1-|z|^{2}}}{\left|1-\bar{\tau}_{0} \tau+\left(\bar{\tau}_{0}-\bar{z}\right) \tau\right|} \leq \frac{\sqrt{1-|z|^{2}}}{\left|\tau_{0}-\tau\right|-\left|\tau_{0}-z\right|} \leq \frac{2}{\delta} \sqrt{1-|z|^{2}} .
$$

Therefore $\left\|\eta_{1} g_{1} k_{z}\right\|_{2} \rightarrow 0$ as $z \rightarrow \tau_{0}$ in $D$. Thus (2.5) follows from (2.6) and (2.7).

Proposition 8. $2\left\|\Phi_{\tau}\left(H_{g}^{*} H_{f}\right)\right\| \geq P_{2}(Q f, Q g ; \tau)$ for all $f, g \in B M O$ and $\tau \in T$.

Proof. We know that $\left\|H_{g}^{*} H_{f}-T_{\xi_{z}}^{*} H_{g}^{*} H_{f} T_{\xi_{z}}\right\|=\left\|H_{g} k_{z}\right\|_{2}\left\|H_{f} k_{z}\right\|_{2}$ [10 page 480]. It is well known that $H_{f} k_{z}=(1-P) f k_{z}=(Q f-(Q f)(z)) k_{z}$. Let $A \in \mathcal{K}_{\tau}$. Since $\left|k_{z}\right|^{2}=P_{z}$,

$$
\begin{aligned}
2\left\|H_{g}^{*} H_{f}+A\right\| \geq & \left\|\left(H_{g}^{*} H_{f}+A\right)-T_{\xi_{z}}^{*}\left(H_{g}^{*} H_{f}+A\right) T_{\xi_{z}}\right\| \\
\geq & \left(\int_{T}|Q f-(Q f)(z)|^{2} P_{z} d m \int_{T}|Q g-(Q g)(z)|^{2} P_{z} d m\right)^{1 / 2} \\
& \quad-\left\|A-T_{\xi_{z}}^{*} A T_{\xi_{z}}\right\| .
\end{aligned}
$$

By Lemma 7, as $z \rightarrow \tau$ in $D$, the limit superior of the above is $P_{2}(Q f, Q g ; \tau)$.

Lemma 9. There is a $C>0$ such that $L M O(f, g)(\tau) \leq C P_{1}(f, g ; \tau), f, g \in B M O$, $\tau \in T$.

Proof. Given $\tau \in T, f, g \in \mathrm{BMO}$ and $\epsilon>0$, there is a $\delta>0$ such that

$$
P_{1}(f, g ; \tau)+\epsilon \geq \int_{T}|f-f(z)| P_{z} d m \int_{T}|g-g(z)| P_{z} d m \text { if } z \in D \text { and }|z-\tau| \leq \delta .
$$

For each arc $I=\left\{e^{i t}: c-\alpha \leq t \leq c+\alpha\right\}$ with $0<\alpha \leq 1 / 4$, define $z_{I}=(1-\alpha) e^{i c}$. Then

$$
P_{z_{I}}\left(e^{i t}\right)=\frac{1-(1-\alpha)^{2}}{1-2(1-\alpha) \cos (c-t)+(1-\alpha)^{2}} \geq \frac{2-\alpha}{2(1-\alpha)} \cdot \frac{\alpha}{S(c-t)^{2}+\alpha^{2}} \geq \frac{\alpha^{-1}}{1+S}
$$

if $|t-c| \leq \alpha$, where $S=\sup _{0<|v| \leq \pi} v^{-2}(1-\cos v)$. That is, $P_{z_{I}} \geq(\pi(1+S)|I|)^{-1} \chi_{I}$. Note that $\int_{I}\left|f-f_{I}\right| d m /|I| \leq 2 \int_{I}\left|f-f\left(z_{I}\right)\right| d m /|I|$. Clearly, there is a $\sigma>0$ such that if $I$ has the property that $|\lambda-\tau| \leq \sigma$ for every $\lambda \in I$, then $\left|z_{I}-\tau\right| \leq \delta$. Thus, for such an $I$, since $\left|z_{I}-\tau\right| \leq \delta$, we have

$$
\begin{aligned}
P_{1}(f, g ; \tau)+\epsilon & \geq\{\pi(1+S)\}^{-2} \cdot \frac{1}{|I|} \int_{I}\left|f-f\left(z_{I}\right)\right| d m \frac{1}{|I|} \int_{I}\left|g-g\left(z_{I}\right)\right| d m \\
& \geq\{2 \pi(1+S)\}^{-2} \cdot \frac{1}{|I|} \int_{I}\left|f-f_{I}\right| d m \frac{1}{|I|} \int_{I}\left|g-g_{I}\right| d m .
\end{aligned}
$$

Since $\epsilon>0$ is arbitrary, this implies $\{2 \pi(1+S)\}^{2} P_{1}(f, g ; \tau) \geq \operatorname{LMO}(f, g)(\tau)$.

Lemma 10. Let $f \in B M O$. If $\left\{I_{n}\right\}$ is a sequence of arcs in $T$ such that

$$
\lim _{n \rightarrow \infty} \frac{1}{\left|I_{n}\right|} \int_{I_{n}}\left|f-f_{I_{n}}\right| d m=0,
$$

then, for every $p \in[1, \infty)$, we have

$$
\lim _{n \rightarrow \infty} \frac{1}{\left|I_{n}\right|} \int_{I_{n}}\left|f-f_{I_{n}}\right|^{p} d m=0 .
$$


Proof. The John-Nirenberg theorem [3, page 230] tells us that

$$
\left|\left\{\tau \in I:\left|f(\tau)-f_{I}\right|>\lambda\right\}\right| /|I| \leq A \exp \left(-B \lambda /\|f\|_{\mathrm{BMO}}\right)
$$

if $|I|>0$ and $\lambda>0$, where $A>0$ and $B>0$ are absolute constants. Let $K \in \mathbf{N}$ be given. Writing $I_{n}=E_{n, K} \cup\left(\bigcup_{k \geq K} F_{n, k}\right)$ with $E_{n, K}=\left\{\tau \in I_{n}:\left|f(\tau)-f_{I}\right| \leq K\right\}$ and $F_{n, k}=\left\{\tau \in I_{n}: k<\left|f(\tau)-f_{I}\right| \leq k+1\right\}$, we have

$$
\begin{aligned}
& \frac{1}{\left|I_{n}\right|} \int_{I_{n}}\left|f-f_{I_{n}}\right|^{p} d m=\frac{1}{\left|I_{n}\right|} \int_{E_{n, K}}\left|f-f_{I_{n}}\right|^{p} d m+\sum_{k=K}^{\infty} \frac{1}{\left|I_{n}\right|} \int_{F_{n, k}}\left|f-f_{I_{n}}\right|^{p} d m \\
& \quad \leq \frac{K^{p-1}}{\left|I_{n}\right|} \int_{E_{n, K}}\left|f-f_{I_{n}}\right| d m+A \sum_{k=K}^{\infty}(k+1)^{p} \exp \left(-B k /\|f\|_{\text {BMO }}\right) .
\end{aligned}
$$

The assumption $\lim _{n \rightarrow \infty} \int_{I_{n}}\left|f-f_{I_{n}}\right| d m /\left|I_{n}\right|=0$ now yields

$$
\limsup _{n \rightarrow \infty} \frac{1}{\left|I_{n}\right|} \int_{I_{n}}\left|f-f_{I_{n}}\right|^{p} d m \leq A \sum_{k=K}^{\infty}(k+1)^{p} \exp \left(-B k /\|f\|_{\text {BMO }}\right) .
$$

Since $K \in \mathbf{N}$ is arbitrary, this completes the proof.

Proof of Theorem 6. We first prove that (b) implies (d). By the circular symmetry, it suffices to consider the case $\tau=1$. Let $f, g \in \mathrm{BMO}$ be such that $M_{p}(f, g ; 1)=0$. Let $0<\alpha<1 / 64$ and $N=N(\alpha) \in \mathbf{N}$ be such that $\alpha^{1 / 4} \leq 2^{-N}<2 \alpha^{1 / 4}$. For each $z=r_{z} e^{i \theta_{z}}$ with $r_{z} \in[0,1)$ and $\theta_{z} \in(-\pi, \pi]$, define $J_{z}=\left\{e^{i t}: t \in\left(\theta_{z}-\pi, \theta_{z}+\pi\right]\right.$, $\left.\left|t-\theta_{z}\right| \leq 2^{N}\left(1-r_{z}\right)\right\}$. There is an $\eta \in(0,1 / 2)$ such that

$$
\frac{1}{\left|J_{z}\right|} \int_{J_{z}}\left|f-f_{J_{z}}\right|^{p} d m \frac{1}{\left|J_{z}\right|} \int_{J_{z}}\left|g-g_{J_{z}}\right|^{p} d m \leq \alpha \text { if }|1-z| \leq \eta \text { and } z \in D .
$$

Hence for any $z \in D$ with $|1-z| \leq \eta$, one of the factors on the left must not exceed $\sqrt{\alpha}$.

Let $w \in D$ with $|1-w| \leq \eta$ be given. Without loss of generality, let us assume $\int_{J_{w}}\left|f-f_{J_{w}}\right|^{p} d m /\left|J_{w}\right| \leq \sqrt{\alpha}$. Define $I_{w, n}=\left\{e^{i t}: t \in\left(\theta_{w}-\pi, \theta_{w}+\pi\right],\left|t-\theta_{w}\right| \leq\right.$ $\left.2^{n}\left(1-r_{w}\right)\right\}$ and $J_{w, n+1}=I_{w, n+1} \backslash I_{w, n}, n \geq N$. Because $|a+b|^{p} \leq 2^{p-1}\left(|a|^{p}+|b|^{p}\right)$, we have

$$
\begin{aligned}
& 2^{-p} \int_{T}|f-f(w)|^{p} P_{w} d m \leq \frac{1}{2} \int_{T}\left|f-f_{J_{w}}\right|^{p} P_{w} d m+\frac{1}{2}\left|f_{J_{w}}-f(w)\right|^{p} \\
& \quad \leq \int_{T}\left|f-f_{J_{w}}\right|^{p} P_{w} d m \\
& \quad=\int_{J_{w}}\left|f-f_{J_{w}}\right|^{p} P_{w} d m+\sum_{n=N+1}^{\infty} \int_{J_{w, n}}\left|f-f_{J_{w}}\right|^{p} P_{w} d m=t+\sum_{n=N+1}^{\infty} t_{n} .
\end{aligned}
$$

Now $\left\|P_{w}\right\|_{\infty}=\left(1-r_{w}^{2}\right) /\left(1-r_{w}\right)^{2} \leq 2 /\left(1-r_{w}\right)=2^{N+1} / 2^{N}\left(1-r_{w}\right)=2^{N+1} / \pi\left|J_{w}\right| \leq$ $2 \alpha^{-1 / 4} / \pi\left|J_{w}\right|$ by the definition of $N$. Therefore

$$
t \leq \frac{2 \alpha^{-1 / 4}}{\pi\left|J_{w}\right|} \int_{J_{w}}\left|f-f_{J_{w}}\right|^{p} d m \leq \alpha^{-1 / 4} \sqrt{\alpha}=\alpha^{1 / 4} .
$$


Let $s=\inf _{0<|v|<\pi} v^{-2}(1-\cos v)$. Then $P_{w}\left(e^{i t}\right) \leq\left(1-r_{w}^{2}\right) / s\left(t-\theta_{w}\right)^{2}$ since $1 / 2 \leq$ $r_{w}<1$. Thus, if $\left|t-\theta_{w}\right|>2^{n-1}\left(1-r_{w}\right)$, then $P_{w}\left(e^{i t}\right) \leq 4\left(1-r_{w}^{2}\right) / 2^{2 n} s\left(1-r_{w}\right)^{2} \leq$ $2^{3-n}\left\{2^{n} s\left(1-r_{w}\right)\right\}^{-1}$. Since $\left|I_{w, n}\right| \leq 2^{n}\left(1-r_{w}\right) / \pi$, we have

$$
\begin{aligned}
t_{n} & \leq \frac{2^{3-n}}{2^{n}\left(1-r_{w}\right) s} \int_{J_{w, n}}\left|f-f_{J_{w}}\right|^{p} d m \\
& \leq \frac{2^{2-n+p}}{s \pi\left|I_{w, n}\right|} \int_{I_{w, n}}\left\{\left|f-f_{I_{w, n}}\right|^{p}+\left|f_{I_{w, n}}-f_{J_{w}}\right|^{p}\right\} d m .
\end{aligned}
$$

There is an absolute constant $C>0$ such that $\left|f_{J_{w}}-f_{I_{w, n}}\right| \leq(n-N) C\|f\|_{\text {BMO }}$ for all $n \geq N+1$ [3, Lemma VI.1.1]. Hence $t_{n} \leq\left(2^{2-n+p} / s \pi\right)\left\{A_{p}^{p}+(C n)^{p}\right\}\|f\|_{\mathrm{BMO}}^{p}$, where $A_{p}$ depends only on $p$ [3, page 233]. Combining this with (2.8), (2.9) and (2.3), we obtain

$$
\int_{T}|f-f(w)|^{p} P_{w} d m \int_{T}|g-g(w)|^{p} P_{w} d m \leq B(p)\left\{\alpha^{1 / 4}+\sum_{n=N+1}^{\infty} 2^{-n} n^{p} U^{p}\right\} U^{p}
$$

if $|1-w| \leq \eta$ and $w \in D$, where $B(p)$ depends only on $p$ and $U=\max \left\{\|f\|_{\text {BMO }}\right.$, $\|g\|_{\text {BMO }}$. Since $2^{-N} \leq 2 \alpha^{1 / 4}$, this shows that $P_{p}(f, g ; 1)=0$. Hence (b) implies (d).

Next we prove that (a) implies (b). Assuming the contrary, we would have a $p \in(1, \infty)$ and a sequence $\left\{I_{n}\right\}$ of arcs such that $\lim _{n \rightarrow \infty} \sup \left\{|\sigma-\tau|: \sigma \in I_{n}\right\}=0$ and such that

$$
\lim _{n \rightarrow \infty} \frac{1}{\left|I_{n}\right|} \int_{I_{n}}\left|f-f_{I_{n}}\right|^{p} d m \frac{1}{\left|I_{n}\right|} \int_{I_{n}}\left|g-g_{I_{n}}\right|^{p} d m=\left(M_{p}(f, g ; \tau)\right)^{p}>0 .
$$

Now $\int_{I}\left|h-h_{I}\right|^{p} d m /|I| \leq\left(A_{p}\|h\|_{\mathrm{BMO}}\right)^{p}$, where $A_{p}>0$ depends only on $p$, page 233]. And the condition $M_{1}(f, g ; \tau)=0$ implies

$$
\lim _{n \rightarrow \infty} \min \left\{\frac{1}{\left|I_{n}\right|} \int_{I_{n}}\left|f-f_{I_{n}}\right| d m, \frac{1}{\left|I_{n}\right|} \int_{I_{n}}\left|g-g_{I_{n}}\right| d m\right\}=0 .
$$

Thus (2.10) contradicts Lemma 10. This proves that (a) implies (b).

It is trivial that (d) implies (c). Lemma 9 tells us that (c) implies (a). This completes the proof.

Proof of Theorem 2. (a): Suppose that $H_{g}^{*} H_{f} \in \mathcal{K}_{\tau}$. Then Proposition 8 tells us that $P_{2}(Q f, Q g ; \tau)=0$. Thus $P_{1}(Q f, Q g ; \tau)=0$ by Hölder's inequality. It then follows from Lemma 9 that $M_{1}(Q f, Q g ; \tau)=0$. Now assume that $M_{1}(Q f, Q g ; \tau)=$ 0 . By Theorem 6 , we have $P_{p}(Q f, Q g ; \tau)=0$ for every $p>2$. Hence $H_{g}^{*} H_{f}=$ $H_{Q g}^{*} H_{Q f} \in \mathcal{K}_{\tau}$ by Theorem 5 .

(b): If $\operatorname{LMO}(f, g)(\tau)=0$, then Theorem 6 yields $P_{p}(f, g ; \tau)=0$ for every $p>2$, which imples $H_{g}^{*} H_{f} \in \mathcal{K}_{\tau}$ (Theorem 5). On the other hand, if $H_{g}^{*} H_{f} \in \mathcal{K}_{\tau}$, then $P_{2}(Q f, Q g ; \tau)=0$ by Proposition 8. For a real-valued $h \in L^{2}$, we have $h-h(z)=2 \operatorname{Re}\{Q h-(Q h)(z)\}$. Since $f, g$ are now assumed to be real valued, $P_{2}(Q f, Q g ; \tau)=0$ implies $P_{2}(f, g ; \tau)=0$. Thus $P_{1}(f, g ; \tau)=0$ by Hölder's inequality and $M_{1}(f, g ; \tau)=0$ by Lemma 9 . 


\section{EXPONENTS AND JOINT OSCILLATION}

We will now prove Proposition 4. We start by picking an $R>16$. For each integer $n \geq 100$, let $I_{n}$ denote the interval $\left(2^{-n}-2^{-n} R^{-2}, 2^{-n}\right)$ in $\mathbf{R}$. Define

$$
f\left(e^{i t}\right)=R \sum_{n=100}^{\infty} \chi_{I_{n}}(t) \text { and } g\left(e^{i t}\right)=\chi_{(-\pi / 2,0)}(t),
$$

where $-\pi \leq t<\pi$. Consider the arc $J=\left\{e^{i t}: a<t<b\right\}$, where $|a| \leq 2^{-200}$ and $|b| \leq 2^{-200}$. Now $f$ and $g$ are designed so that $\int_{J}\left|f-f_{J}\right| d m \int_{J}\left|g-g_{J}\right| d m=0$ if either $a \geq 0$ or $b \leq 0$. Assume that $a<0$ and $b>0$. Then there is a $k>100$ such that

$$
2^{-k-1}-2^{-k-1} R^{-2} \leq b<2^{-k}-2^{-k} R^{-2} .
$$

We have $f_{J} \leq R \sum_{n=k+1}^{\infty} R^{-2} 2^{-n} /(b-a) \leq R^{-1} 2^{k+2} \sum_{n=k+1}^{\infty} 2^{-n} \leq 4 R^{-1}$ because $b>2^{-k-2}$ and $a<0$. Thus $|J|^{-1} \int_{J}\left|f-f_{J}\right| d m \leq 2 f_{J} \leq 8 R^{-1}$. Thus, because $\int_{J}\left|g-g_{J}\right| d m /|J| \leq 1$, when $|a| \leq 2^{-200},|b| \leq 2^{-200}$ and $a<b$, we always have

$$
\frac{1}{|J|} \int_{J}\left|f-f_{J}\right| d m \frac{1}{|J|} \int_{J}\left|g-g_{J}\right| d m \leq 8 R^{-1}
$$

regardless of the signs of $a$ and $b$. Consequently $\operatorname{LMO}(f, g)(1) \leq 8 R^{-1}$.

Let $J_{n}=\left\{e^{i t}:|t|<2^{-n}\right\}, n \geq 200$. We next show that

$$
\frac{1}{\left|J_{n}\right|} \int_{J_{n}}\left|f-f_{J_{n}}\right|^{2} d m \frac{1}{\left|J_{n}\right|} \int_{J_{n}}\left|g-g_{J_{n}}\right|^{2} d m \geq \frac{1}{32} .
$$

First of all, $g_{J_{n}}=1 / 2$. Therefore $\int_{J_{n}}\left|g-g_{J_{n}}\right|^{2} d m /\left|J_{n}\right|=1 / 4$. We know from the last paragraph that $f_{J_{n}} \leq 4 R^{-1}<R / 2$. Hence $\left|f-f_{J_{n}}\right|^{2} \geq R^{2} / 4$ on the arc $\tilde{I}_{n}=\left\{e^{i t}: t \in I_{n}\right\}$. Since $\left|\tilde{I}_{n}\right|=\left(2 \pi R^{2} 2^{n}\right)^{-1}$ and $\left|J_{n}\right|=2 \cdot 2^{-n} / 2 \pi$, we have $\int_{J_{n}}\left|f-f_{J_{n}}\right|^{2} d m /\left|J_{n}\right| \geq 1 / 8$. This verifies (3.1).

Let $z_{n}=1-2^{-n}, n \geq 200$. We showed $P_{z_{n}} \geq\left(\pi(1+S)\left|J_{n}\right|\right)^{-1} \chi_{J_{n}}$ in the proof of Lemma 9, where $S=\sup _{0<|v| \leq \pi} v^{-2}(1-\cos v)$. Let $c=\{\pi(1+S)\}^{-1}$. By (3.1),

$$
\begin{aligned}
\int_{T} \mid f & -\left.f\left(z_{n}\right)\right|^{2} P_{z_{n}} d m \int_{T}\left|g-g\left(z_{n}\right)\right|^{2} P_{z_{n}} d m \\
& \geq \frac{c^{2}}{\left|J_{n}\right|^{2}} \int_{J_{n}}\left|f-f\left(z_{n}\right)\right|^{2} d m \int_{J_{n}}\left|g-g\left(z_{n}\right)\right|^{2} d m \\
& \geq \frac{c^{2}}{16} \cdot \frac{1}{\left|J_{n}\right|} \int_{J_{n}}\left|f-f_{J_{n}}\right|^{2} d m \frac{1}{\left|J_{n}\right|} \int_{J_{n}}\left|g-g_{J_{n}}\right|^{2} d m \geq \frac{c^{2}}{512} .
\end{aligned}
$$

Since $z_{n} \rightarrow 1$ as $n \rightarrow \infty$, this implies $P_{2}(f, g ; 1) \geq c / 16 \sqrt{2}$. Proposition 8 now tells us that $\left\|\Phi_{1}\left(H_{g}^{*} H_{f}\right)\right\| \geq P_{2}(Q f, Q g ; 1) / 2 \geq P_{2}(f, g ; 1) / 8 \geq c / 128 \sqrt{2}$, where the second $\geq$ holds because $f$ and $g$ are real-valued functions. Since $\operatorname{LMO}(f, g)(1) \leq$ $8 R^{-1}$ and $R>16$ is otherwise arbitrary, this completes the proof of Proposition 4.

Remark. Given $1 \leq p^{\prime}<p<\infty$ and $\epsilon>0$, one can easily modify the above construction to obtain a pair of $f, g \in L^{\infty}$ such that

$$
0<\sup _{I}\left(\frac{1}{|I|} \int_{I}\left|f-f_{I}\right|^{p^{\prime}} d m \frac{1}{|I|} \int_{I}\left|g-g_{I}\right|^{p^{\prime}} d m\right)^{1 / p^{\prime}} \leq \epsilon M_{p}(f, g ; 1) .
$$


In fact one can keep the same $g$ and simply let

$$
f\left(e^{i t}\right)=R \sum_{n=100}^{\infty} \chi_{\left(2^{-n}-2^{-n} R^{-p}, 2^{-n}\right)}(t), \quad-\pi \leq t<\pi,
$$

with a sufficiently large $R$.

\section{REFERENCES}

[1] S. Axler, S.-Y. Chang, and D. Sarason, Products of Toeplitz operators, Integral Eq. Operator Theory 1 (1978), 285-309. MR 80d:47039

[2] R. Douglas, Banach algebra techniques in operator theory, Academic Press, New York, 1972. MR 50:14335

[3] J. Garnett, Bounded analytic functions, Academic press, New York, 1981. MR 83g:30037

[4] P. Muhly and J. Xia, Calderon-Zygmund operators, local mean oscillation and certain automorphisms of the Toeplitz algebra, Amer. J. Math. 117 (1995), 1157-1201. MR 96k:47045

[5] D. Sarason, Functions of vanishing mean oscillation, Trans. Amer. Math. Soc. 207 (1975), 391-405. MR 51:13690

[6] D. Sarason, Products of Toeplitz operators, Lecture Notes in Mathematics Vol. 1573, SpringerVerlag, Berlin, 1994.

[7] A. Volberg, Two remarks concerning the theorem of S. Axler, S.-Y. A. Chang, and D. Sarason, J. Operator Theory 8 (1982), 209-218. MR 84h:47038a

[8] D. Xia and D. Zheng, Products of Hankel operators, Integral Eq. Operator Theory 29 (1997), 339-363. CMP 98:03

[9] J. Xia, Rigged non-tangential maximal functions associated with Toeplitz operators and Hankel operators, Pacific J. Math. 182 (1998), 385-396. CMP 98:09

[10] D. Zheng, The distribution function inequality and products of Toeplitz operators and Hankel operators, J. Funct. Anal. 138 (1996), 477-501. MR 97e:47040]

[11] D. Zheng, Toeplitz operators and Hankel operators on the Hardy space of the unit sphere, J. Funct. Anal. 149 (1997), 1-24. MR 98m:47038

Department of Mathematics, State University of New York at Buffalo, Buffalo, NEW YoRK 14214

E-mail address: jxia@acsu.buffalo.edu 